See end of article for authors' affiliations

Correspondence to: Dr Paul R Jarman, National Hospital for Neurology and Neurosurgery, Queen Square London WCIN 3BG, UK: paul.jarman@uclh.org

\section{HUNTINGTON'S DISEASE}

Huntington's disease (HD) is the prototypic neurogenetic disorder, one of the first to be mapped (1983) and subsequently cloned (1993), and the model on which presymptomatic genetic testing is based.

The clinical triad of movement disorder, psychiatric features, and eventual dementia will be well known to neurologists. Chorea is the first manifestation in about two thirds of patients, initially a mild fidgetiness apparent only to the careful observer, which gradually progresses and may be the only clinical manifestation of HD for several years. Severe chorea may respond well to neuroleptics such as sulpiride. Personality change and eye movement disorders including slow saccades, and head thrusting or blinking to generate saccadic eye movements, are also common early features. A wide range of movement disorders including parkinsonism, loss of postural stability, and dystonia eventually supervene, leading to increasingly functional impairment. Progressive weight loss, often resulting in cachexia, is common. Presentation is usually in the forth or fifth decades, but may be at almost any age. The juvenile onset form of HD may present with parkinsonism, the so-called Westphal variant, while late onset forms may cause chorea alone.

Expansion of a polyglutamine (CAG) trinucleotide repeat beyond the critical threshold of 36 repeats results in disease, and forms the basis of the polymerase chain reaction based genetic test. Expansion size is inversely related to age at onset, but the range in age at onset for a given repeat size is so large that repeat size is not a useful predictor for individuals. Inheritance is dominant with full penetrance, meaning that almost all mutation carriers will eventually develop the disease, except those with 36-39 repeats where penetrance is reduced. Meiotic instability with a tendency to increasing expansion size, particularly during spermatogenesis, provides the molecular basis for the phenomenon of anticipation. Thus, juvenile onset cases with very large expansions usually have an affected father. A small minority $(<1 \%)$ of individuals have expansions in an intermediate range (29-35 repeats), believed to be an asymptomatic but unstable pre-mutation range, which may expand to cause disease in offspring. Dentatorubropallidoluysian atrophy (DRPLA), also caused by a trinucleotide repeat expansion, may be difficult to distinguish clinically from early onset HD, and so is usually tested for with HD.

Predictive genetic testing of asymptomatic at-risk relatives of affected patients is governed by international guidelines (see Hanna and Wood on page ii2). Prenatal testing in known mutation carriers is routinely available, while linkage based exclusion testing is available to those at-risk women who do not wish to know their own gene status. The latter depends on termination of a pregnancy where linkage shows the fetus to have the same $50 \%$ genetic risk as the mother. Preimplantation diagnosis based on selection of unaffected IVF embryos is available in some centres.

\section{THE DYSTONIAS}

Dystonia is a feature of many hereditary neurodegenerative and metabolic disorders. In most of these disorders, dystonia usually occurs in the setting of a broader clinical phenotype, and careful clinical assessment reveals other features such as cognitive involvement, pyramidal signs, or ocular abnormalities. 


\section{Primary dystonias}

In this group of disorders, dystonia or dystonic tremor is (with the exception of parkinsonism in dopa responsive dystonia (DRD)) the only clinical manifestation, and imaging and pathology are usually unrevealing. These disorders have a genetic basis, with dominant inheritance in most cases. The most important of these disorders to the neurologist are DYTl dystonia and DRD.

\section{DYT1 dystonia}

Mutations in the DYTl gene, encoding the protein torsin A, are responsible for the most common form of primary dystonia, inherited as an autosomal dominant trait, with low (30\%) penetrance, and variable expression. The typical phenotype consists of childhood limb onset dystonia, which gradually spreads over subsequent years to become generalised, affecting trunk and limb muscles. The foot is usually the first body part affected, and the craniocervical muscles are typically spared, even with severe disease. Disease severity may vary widely from focal or segmental dystonia to severe generalised dystonia even within a single family, while most mutation carriers are asymptomatic.

Genetic testing is possible by screening for deletion of one of a pair of GAG (glutamic acid) residues in the DYTl gene, which accounts for most cases. Diagnostic testing is worthwhile in patients with dystonia starting before the age of 26 , or in older patients with a family history of early onset dystonia. As well as allowing genetic counselling, a positive test spares patients from extensive investigation for other causes of dystonia. Approximately $60 \%$ of cases with the typical phenotype are mutation positive in the UK population, indicating the existence of other, as yet unmapped dystonia genes, and non-genetic phenocopies. The gene is particularly prevalent in the Ashkenazi Jewish population due to a founder mutation estimated to have occurred about 400 years ago.

Oral treatments for dystonia are often unrewarding, although anticholinergics, often at high doses, may be beneficial in some patients. Recent experience with GPi deep brain stimulation has been extremely encouraging, with notable improvements seen in most patients reported.

\section{Dopa responsive dystonia}

Although rare, the importance of DRD lies in its dramatic and sustained response to levodopa. The clinical presentation is similar to DYT-1 dystonia, with childhood limb onset dystonia and gradual progression to generalised dystonia, the only specific clues being a diurnal variation in symptoms with improvement after sleep, and mild parkinsonism in some patients. A therapeutic trial of levodopa (Sinemet $275 \mathrm{mg}$ three times daily for three months) is therefore mandatory in all patients with dystonia starting before the age of 30 years. Atypical presentations of DRD, including a spastic paraparesis-like presentation and apparent cerebral palsy are well described and should prompt a trial of levodopa.

Mutations in the gene encoding the GTP cyclohydrolase 1 enzyme $(\mathrm{GCHl})$ cause DRD. This enzyme catalyses the initial and rate limiting step in the synthesis of tetrahydrobiopterin, which is an essential co-factor for tyrosine hydroxylase in the dopamine synthesis pathway. Loss of enzyme activity therefore results in dopamine depletion. The rare autosomal recessive form of DRD may be caused by mutations in the tyrosine hydroxylase gene.

The diagnosis of DRD is based on the clinical picture and dramatic response to levodopa. Genetic screening for mutations is not routinely available since most mutations are "pri-

\section{Abbreviations}

ADCA: autosomal dominant cerebellar ataxia

AR-JP: autosomal recessive juvenile parkinsonism

AT: ataxia telangiectasia

CTX: cerebrotendinous xanthomatosis

DRD: dopa responsive dystonia

DRPLA: dentatorubropallidoluysian atrophy

FA: Friedreich's ataxia

HD: Huntington's disease

OMIM: Online Mendelian Inheritance in Man

PDC: paroxysmal dystonic choreathetosis

PKC: paroxysmal kinesigenic choreoathetosis

PSP: progressive supranuclear palsy

SCA: spino-cerebellar ataxia

vate". Patients with DRD are unable to rapidly excrete an oral phenylalanine challenge (tetrahydrobiopterin is also a cofactor for phenylalanine hydroxylase), and this is the basis for the phenylalanine loading test that may be helpful in equivocal cases.

\section{Paroxysmal dyskinesias}

These disorders have been classified with the dystonias, but in fact they cause a mixed movement disorder with elements of dystonia, chorea, and ballism during attacks. Paroxysmal kinesigenic choreoathetosis (PKC) is characterised by short lived attacks, usually lasting only a few seconds, but occurring up to 100 times per day. Attacks are usually precipitated by sudden movement or the anticipation of movement. It responds dramatically to low doses of anticonvulsants and is usually dominantly inherited with reduced penetrance. Linkage is to contiguous loci on chromosome 16, but no gene has yet been identified. Infantile convulsions are now recognised as a phenotypic manifestation of PKC in many families.

In contrast, paroxysmal dystonic choreathetosis (PDC) is a rarer disorder with less frequent, more prolonged attacks (hours in duration), usually precipitated by alcohol, caffeine or stress. Inheritance is dominant with high penetrance, meaning that most patients have affected relatives. Since investigations and examination between attacks are normal, many patients are labelled as non-organic.

\section{ESSENTIAL TREMOR}

Up to $70 \%$ of patients with essential tremor have affected relatives, and several dominantly inherited genes are believed to exist. Two loci have been mapped (chromosomes 2 and 3) but no genes yet identified.

\section{WILSON'S DISEASE}

Mutations in the gene coding for a copper transporting ATPase lead to impaired biliary copper excretion and subsequent accumulation in the liver, brain, kidney, and cornea.

Presentation in younger patients is usually with liver disease or haemolytic anaemia, while adults more commonly present with neurological and psychiatric symptoms. There is usually established cirrhosis by the time neurological features develop, and many patients give a history of previous episodes of unexplained hepatitis or haemolytic anaemia. Orofacial dystonia causing dysarthria, dysphagia, and drooling (a sort of pseudo pseudobulbar palsy) is common, as is dystonic posturing, tremor, and parkinsonism. Subtle personality change is often present, and more obvious neuropsychiatric disturbances including psychosis may lead to initial psychiatric 
Table 1 Availability of genetic testing for movement disorders and ataxias in the UK

\begin{tabular}{ll}
\hline Disease/test & Laboratory \\
\hline HD expansion & $\begin{array}{l}\text { Most regional genetics labs } \\
\text { DRPLA }\end{array}$ \\
Dystonia - DYT1 deletion & $\begin{array}{l}\text { Neurogenetics lab, Queen Square, } \\
\text { London }\end{array}$ \\
Dystonia-DRD & $\begin{array}{l}\text { Under development, Queen Square, } \\
\text { London }\end{array}$ \\
Ataxia-SCA 1,2,3,6,7 & Most regional genetics labs \\
Freidreich's ataxia & $\begin{array}{l}\text { Most regional genetics labs } \\
\text { Sequencing for point mutations in }\end{array}$ \\
Parkin & $\begin{array}{l}\text { Liverpool } \\
\text { Under development, Queen Square, } \\
\text { London }\end{array}$ \\
Wilson's disease & Sheffield \\
Mitochondrial mutations & Neurogenetics lab, Queen Square, \\
& London
\end{tabular}

referral. Young adults presenting with any movement disorder, with the exception of tics, should prompt exclusion of Wilson's disease, but the neurologist should be aware that patients may exceptionally present in their 50s.

A Kayser-Fleisher ring is said to be invariably present in neurological Wilson's disease, but slit lamp examination may be needed to detect it. Mild reductions in caeruloplasmin concentrations may be seen in some asymptomatic carriers (up to $1 \%$ of the population), so liver biopsy to measure copper content, or radiolabelled copper studies are sometimes required. Genetic studies, either gene sequencing to identify mutations or linkage analysis to track disease chromosomes, may be particularly useful in screening siblings of affected patients. Treatment with copper chelating agents should be supervised in specialist clinics.

\section{NEUROACANTHOCYTOSIS}

Chorea and orofacial dyskinesias with tics and lip/tongue biting occur in association with neuropathy, seizures, psychiatric, and cognitive involvement in this multi-system disorder characterised by the presence of acanthocytes in fresh blood films. Recessively inherited mutations in the chorein gene on chromosome 9, or X linked mutations in the Kell blood group protein, may both cause indistinguishable phenotypes.

\section{PARKINSON'S DISEASE}

Although Parkinson's disease is usually sporadic, several genes have been identified for the rarer inherited forms of the disease (at the time of writing 10 "PARK" loci have been assigned). The autosomal recessive forms may account for a proportion of patients with early onset, apparently sporadic disease, since the inheritance pattern may not be apparent where only one sibling is affected. At present routine genetic testing is not yet available for any of these genes.

\section{PARK1 ( $\alpha$-synuclein)}

Mutations in the $\alpha$-synuclein gene are a very rare cause of dominantly inherited Lewy body-positive Parkinson's disease. The demonstration that $\alpha$-synuclein is the major component of Lewy bodies means that this locus is of considerable scientific interest but of little clinical relevance.

\section{PARK2 (Parkin)}

Autosomal recessive juvenile parkinsonism (AR-JP) was originally described in young Japanese patients with a slowly progressive form of parkinsonism characterised by excellent levodopa responsiveness, early dystonia and dyskinesias, and hyperreflexia. Following identification of pathogenic mutations in the parkin gene, the clinical spectrum has been expanded to include late onset, tremor dominant parkinsonism, indistinguishable from typical Parkinson's disease. It is now known to occur worldwide as the most common familial form of Parkinson's disease, accounting for three quarters of apparently sporadic cases with onset before 20 years, a quarter of cases with onset between 20-30 years, and 3-7\% of cases with onset before age 50 . Ages at onset into the 70s have been described. Parkin is a ubiquitin-protein ligase enzyme (E3) involved in protein degradation.

The PARK6 locus on chromosome 1 has not yet been fully characterised, but early indications are that this locus may also be an important cause of autosomal recessive Parkinson's disease.

\section{Other parkinsonian disorders}

Other genetic disorders may occasionally present with parkinsonism, including tau mutations which may sometimes cause a progressive supranuclear palsy (PSP)-like presentation, SCA3 mutations which may present with parkinsonism as well as ataxia, the juvenile presentation of HD, and Wilson's disease. Both PSP and corticobasalganglionic degeneration are strongly associated with a specific haplotype around the tau gene, although neither disorder is overtly familial.

\section{THE ATAXIAS}

A simple clinical point when considering the nature of a possible inherited ataxia is the age of onset. As a general rule early onset ( $<20$ years) tends to be autosomal recessive, later onset ( $>25$ years) is usually autosomal dominant, and X linked inheritance is very rare.

\section{Early onset ataxias}

The default position of an ataxia presenting earlier than the mid 20s is: "could this be Friedreich's ataxia?" This is far and away the most common disease, classically presenting with gait ataxia, but with a number of additional features including dysarthria, and pyramidal tract involvement. Initially this latter feature may be mild, with just extensor plantar responses, but almost invariably a pyramidal pattern of weakness, sometimes leading to paralysis, in the legs occurs. A peripheral neuropathy is seen with absent reflexes, large fibre sensory abnormalities, and occasionally distal wasting, particularly in the upper limbs. Skeletal abnormalities are also commonly found including scoliosis and pes cavus. Additionally optic atrophy and deafness may be found. Nystagmus is seen in only about $20 \%$, but the extraocular movements are nearly always abnormal, with broken pursuit, dysmetric saccades, and square wave jerks.

Investigation of patients reveals an axonal sensory neuropathy; an abnormal ECG with widespread T wave inversion. Diabetes mellitus occurs in 10\% of patients with Friedreich's ataxia (FA), and a further $10-20 \%$ have impaired glucose tolerance. Therefore yearly screening involves ECG and serum glucose with a low threshold for referral for further specialist advice.

The gene frataxin was cloned in 1996. The predominant mutation is a trinucleotide repeat (GAA) in intron 1 of this gene. Expansion of both alleles is found in over $96 \%$ of patients. The remaining patients have point mutations in the frataxin gene. The DNA test for the repeat is relatively simple and widely available (table 1). The length of the repeat is a determinant of age of onset and therefore to some degree 
Table 2 Autosomal dominant cerebellar ataxia. Only those for which gene tests are currently available are listed

\begin{tabular}{|c|c|c|c|c|}
\hline ADCA type & Clinical features & $\begin{array}{l}\text { Genetic loci and } \\
\text { chromosomal location }\end{array}$ & $\begin{array}{l}\text { Normal } \\
\text { allele } \\
\text { (repeats) }\end{array}$ & $\begin{array}{l}\text { Pathological } \\
\text { allele (repeats) }\end{array}$ \\
\hline ADCA I & $\begin{array}{l}\text { Cerebellar syndrome plus: } \\
\text {-pyramidal signs } \\
\text {-supranuclear ophthalmoplegia } \\
\text {-extrapyramidal signs } \\
\text {-peripheral neuropathy } \\
\text {-dementia }\end{array}$ & $\begin{array}{l}\text { SCA } 16 p 22-23 \\
\text { SCA } 212 q 23-24.1 \\
\text { SCA } 314 q 32.1 \\
\text { SCA } 125 q 31-33 \\
\text { SCA } 176 q 27\end{array}$ & $\begin{array}{l}6-44 \\
13-33 \\
12-40 \\
22-30 \\
25-42\end{array}$ & $\begin{array}{l}39-83 \\
32-77 \\
54-89 \\
66-78 \\
47-63\end{array}$ \\
\hline ADCA ॥ & $\begin{array}{l}\text { Cerebellar syndrome plus: } \\
\text {-pigmentary maculopathy } \\
\text {-other signs as ADCA I }\end{array}$ & SCA 7 3p12-21.1 & $4-35$ & $36-306$ \\
\hline ADCA III & $\begin{array}{l}\text { "Pure" cerebellar syndrome } \\
\text { Mild pyramidal signs }\end{array}$ & SCA 6 19p13 & $4-18$ & $20-33$ \\
\hline
\end{tabular}

influences the severity in that early onset tends to progress more rapidly. To date there are no reports of a patient with FA with two point mutations - that is, there is always at least one expanded repeat. Therefore if FA is suspected then the diagnosis is effectively excluded if two normal sized repeats are seen.

The other autosomal recessive ataxias are individually rare and often have a metabolic abnormality underlying the pathogenesis. If the ataxia starts very early in life consider the sphingomyelin lipidoses, metachromatic leukodystrophy, galactosylceramide lipidosis (Krabbe's disease), and the hexosaminidase deficiencies. Also within this group is adrenoleukomyeloneuropathy, a phenotypic variant of adrenoleukodystrophy. Ataxia may be prominent in Niemann-Pick disease type C.

Cholestanolosis (also called cerebrotendinous xanthomatosis $(\mathrm{CTX}))$ is a rare autosomal recessive disorder caused by defective bile salt metabolism, resulting from a deficiency of mitochondrial sterol 27 hydroxylase. It gives rise to ataxia, dementia, spasticity, peripheral neuropathy, cataracts, and tendon xanthomata in the second decade of life. Treatment with chenodeoxycholic acid appears to improve neurological function.

Various phenotypes that are classifiable as hereditary ataxias have been described in the mitochondrial encephalomyopathies, many of which are associated with a defect of mitochondrial DNA. These include the Kearns-Sayre syndrome. If additional neurological features are present-for example, visual problems, cognitive decline, and myoclonusthen the syndrome of progressive myoclonic ataxia should be considered. This may be caused by ceroid lipofuscinosis, sialidosis, and Unverricht-Lundborg's disease as well as mitochondrial disease.

An important but very rare syndrome not to be missed is ataxia caused by vitamin E deficiency; this can occur secondary to malabsorption but can also be caused by abnormalities in a vitamin E transporting protein. Treatment with vitamin E helps prevent deterioration.

There are a number of rare conditions associated with a reduced capacity to perform excision repair of DNA damaged by ultraviolet light and some chemical carcinogens. The most common is ataxia telangiectasia (AT). Clinically related conditions include xeroderma pigmentosum and Cockayne's syndrome. The gene for AT has now been cloned and is called ATM.

\section{Late onset inherited ataxias}

These are usually autosomal dominant and only rarely due to some of the causes listed above. However, those disorders should be considered if the phenotype is compatible.

The dominant ataxias are a clinically and genetically complex group of neurodegenerative disorders (table 2). Autosomal dominant cerebellar ataxia (ADCA) type I is characterised by a progressive cerebellar ataxia and is variably associated with other extracerebellar neurological features such as ophthalmoplegia, optic atrophy, peripheral neuropathy, and pyramidal and extrapyramidal signs. The presence and severity of these signs is, in part, dependent on the duration of the disease. Mild or moderate dementia may occur but it is usually not a prominent early feature. ADCA type II is clinically distinguished from ADCA type I by the presence of pigmentary macular dystrophy, whereas ADCA type III is a relatively "pure" cerebellar syndrome and generally starts at a later age. This clinical classification is still useful, despite the tremendous improvements in our understanding of the genetic basis, because it provides a framework that can be used in the clinic and helps direct the genetic evaluation.

The genetic loci causing the dominant ataxias are given the acronym SCA (spino-cerebellar ataxia). At the time of publication there are over 20 SCA loci identified. Of these the genes are established for SCAs 1, 2, 3, 6, 7, 10, 12, and 17 . Interestingly the "common" ones are all caused by a similar mutational mechanism, expansion of an exonic CAG repeat. The resultant proteins all possess an expanded polyglutamine tract and there are now at least eight conditions caused by these expansions. Other types of ADCA are exceedingly rare.

\section{Idiopathic degenerative late onset ataxias}

In the absence of a clear family history this is rarely genetic. There are a few reports with one or other of the SCAs or occasional FA but these are very infrequent. The main differential in this group of patients is whether or not it is the cerebellar presentation of multiple system atrophy. A frequent clinical problem is whether to test for the identifiable mutations. We believe that, although very unlikely, we have seen an occasional diagnosis emerge. This of course may have significant genetic consequences for the rest of the family. 


\section{Authors' affiliations}

P R Jarman, N W Wood, National Hospital for Neurology and

Neurosurgery, London, UK

\section{KEY REFERENCES}

ii26
KEY REFERENCES

Brain 2002; 125:695-721.
- An up-to-date and comprehensive review.

2 Valente EM, Warner TT, Jarman PR, et al. The role of DYT1 in primary torsion dystonia in Europe. Brain 1998;121:2335-9.

- Phenotype-genotype study of DYT1 dystonia in Europe.

3 Gasser T. Genetics of Parkinson's disease. J Neurol 2001;248:833-40.

4 Harding AE. Friedreich's ataxia: a clinical and genetic study of 90 families with an analysis of early diagnostic criteria and intrafamilial clustering of clinical features. Brain 1981;104:589-620

5 Harding AE. The hereditary ataxias and related disorders. Edinburgh: Churchill Livingstone, 1984

\section{WORLD WIDE WEB}

\section{Surfing for neurogenetics}

G enetics is the area of medicine most associated with the world wide web. This has stemmed from the acclaimed efforts of the International Human Genome Sequencing Consortium to ensure that the draft sequence of the human genome sequence was available online to the public (http://www.genome.gov/). Moreover, the ease of updating information on the web has made it an important medium for rapidly advancing clinical areas such as neurogenetics. As a result there are many excellent websites pertaining to genetics. I shall highlight those that fulfil some or all of the following criteria that make a good web site-namely: design and layout; accurate and up-to-date information; ease of navigation; and multiple links.

As a starting point, patients and doctors may need to familiarise themselves with common genetic terms and concepts. The National Human Genome Research Institute (NHGRI) has produced a very useful talking glossary (http://www.genome.gov/glossary.cfm) that provides clear definitions backed up with detailed audio explanation from various NHGRI researchers including Francis Collins (leader of the Human Genome Project.) The UK Genetic Interest Group (GIG) has a good education section on their web page (http://www.gig.org.uk/). This includes a comprehensive glossary with easy-to-follow pictures for explaining the different inheritance patterns for genetic diseases. The most impressive web page for the number of educational links alone has to be the Genetics Education Center (GEC) run by the University of Kansas at http://www.kumc.edu/gec/.

Given the clinical diversity of neurogenetic conditions, it is unsurprising that no one web site provides patient information encompassing all neurogenetic conditions. However, there are several offering good lists of links. GIG has contact information and links for virtually all UK support groups dealing with the common and rare neurogenetic diseases. Of these the Charcot Marie Tooth (CMT) UK web site is excellent (http://www.cmt.org.uk/). It is easy to navigate and explains the genetics well and includes a children's section. Internationally, the University of Kansas has a "Genetic and rare conditions" site at http:// www.kumc.edu/gec/support/ that lists links for genetic disease support groups in North America as well as the rest of the world.

For the jobbing neurologist who wants to rapidly ascertain up to date information on the genetics of any neurological condition, the best web site is the Online Mendelian Inheritance in Man (OMIM) database (http://www.ncbi.nlm.nih.gov/Omim/). This is now easier to search through via its incorporation into the Entrez system of the National Center for Biotechnology Information (NCBI) at http:// www.ncbi.nlm.nih.gov/entrez/query.fcgi? $\mathrm{db}=\mathrm{OMIM}$. Any entry usually results in multiple hits in descending order of relevance. For any disease the known genes are listed with other relevant information including clinical features and pathogenesis. The accession numbers of genes form links that allow you to access more detailed information about the gene, including its chromosome location and function. Also useful is a list of the reported allelic variants of the gene with respective phenotypic information. All this information is exhaustively referenced with links to cited articles in the Entrez PubMed database. The OMIM database is also useful for neurological diseases that are genetically complex or where a single causative gene has yet to be identified-for example, Gilles de la Tourette syndrome-and provides information from family and mapping studies. Disadvantages of OMIM are that for some entries the information is not always up to date. Also the updating of entries often consists merely of add-on of information rather than complete revision.

Another useful site for neurologists is at http://www.geneclinics.org/. It is free but requires a one-off registration process. Its GeneReview section offers up to date reviews of many genetic and neurogenetic disorders written by leading researchers. These reviews are accessible by an easy search facility. The rest of the site has interesting pages on genetic counselling as well as information on laboratories performing genetic tests, although they are largely in North America. Information on UK genetic service laboratories is available on the GIG web site.

M M K Muqit

Department of Molecular Pathogenesis, Institute of Neurology, Queen Square, London m.muqit@ion.ucl.ac.uk 